\title{
Doppler echocardiographic evaluation of the normal human fetal heart
}

\author{
LINDSEY D ALLAN, SUNDER K CHITA, WIDAD AL-GHAZALI, \\ DIANE C CRAWFORD, MICHAEL TYNAN
}

From the Departments of Perinatal and Paediatric Cardiology, Guy's Hospital, London

SUMmaRy Pulsed wave Doppler estimates of blood flow velocity were made across the mitral, tricuspid, aortic, and pulmonary valves in a series of 120 normal fetuses (gestational age 16-36 weeks). In 36 of these the data were obtained in all four sites. The maximum and mean velocities were calculated for each valve and these values were plotted against gestational age. There was little change in these values throughout pregnancy. The orifice dimensions of the valves were measured by cross sectional echocardiography. At all ages the tricuspid orifice was larger than the mitral and the pulmonary orifice was larger than the aortic. The blood flow values for each valve were derived from the product of the mean velocity and the valve orifice dimensions. The output of the right ventricle was usually, but not always, greater than that of the left ventricle. Combined ventricular output increased from approximately $50 \mathrm{ml} / \mathrm{min}$ at 18 weeks to $1200 \mathrm{ml} / \mathrm{min}$ at term.

Despite limitations in the accuracy of the technique these results form a useful basis for the analysis of blood flow in the normal fetus and for the interpretation of abnormal Doppler findings in prenatal life.

The anatomy of the fetal heart can be readily examined by echocardiography from as early as 16 weeks' gestation. ${ }^{1}$ Both normal and abnormal structure can be identified. ${ }^{2}{ }^{3}$ Blood flow velocity can now be estimated by Doppler examination in conjunction with cross sectional imaging. The volume of blood flow across each cardiac valve can be derived from the Doppler study. ${ }^{45}$ Previous work on fetal haemodynamic function has centred mainly on the lamb; in this study we report the Doppler evaluation of blood flow in a series of normal human fetuses.

\section{Patients and methods}

Patients were selected at random from pregnant women attending for fetal echocardiography because of a family history of congenital heart disease or attending the antenatal clinic. An initial fetal echocardiogram was performed to ensure that the fetal heart was structurally normal. Patients with any

Requests for reprints to Dr Lindsey D Allan, Department of Perinatal Cardiology, Guy's Hospital, St Thomas Street, London SE1 9RT.

Accepted for publication 12 February 1987 abnormality of pregnancy were excluded from the study. All pregnancies were dated by early estimation of the biparietal diameter or the femoral length or both.

A Hewlett Packard 77020A phased array sector scanner was used with a $5 \mathrm{MHz}$ transducer. Occasionally, in late pregnancy adequate imaging could only be obtained with a $3 \mathrm{MHz}$ transducer. In order to minimise errors in measurement only subjects in whom good quality images were achieved were included in the study. The total ultrasound examination time was limited to 30 minutes per patient. Within this time constraint it was not possible to obtain Doppler velocity traces of optimal quality at all four sites in every patient. Poor quality traces were excluded from analysis. Thus the patients were not consecutive.

Doppler velocity measurements were obtained by placing the sample volume just distal to the atrioventricular valve leaflets in the left or right ventricles and distal to the arterial valve in the aorta or the pulmonary artery. Only an angle of $<20^{\circ}$ between the direction of flow and the direction of the Doppler beam was regarded as acceptable. With our equipment the angle is indicated manually by the 

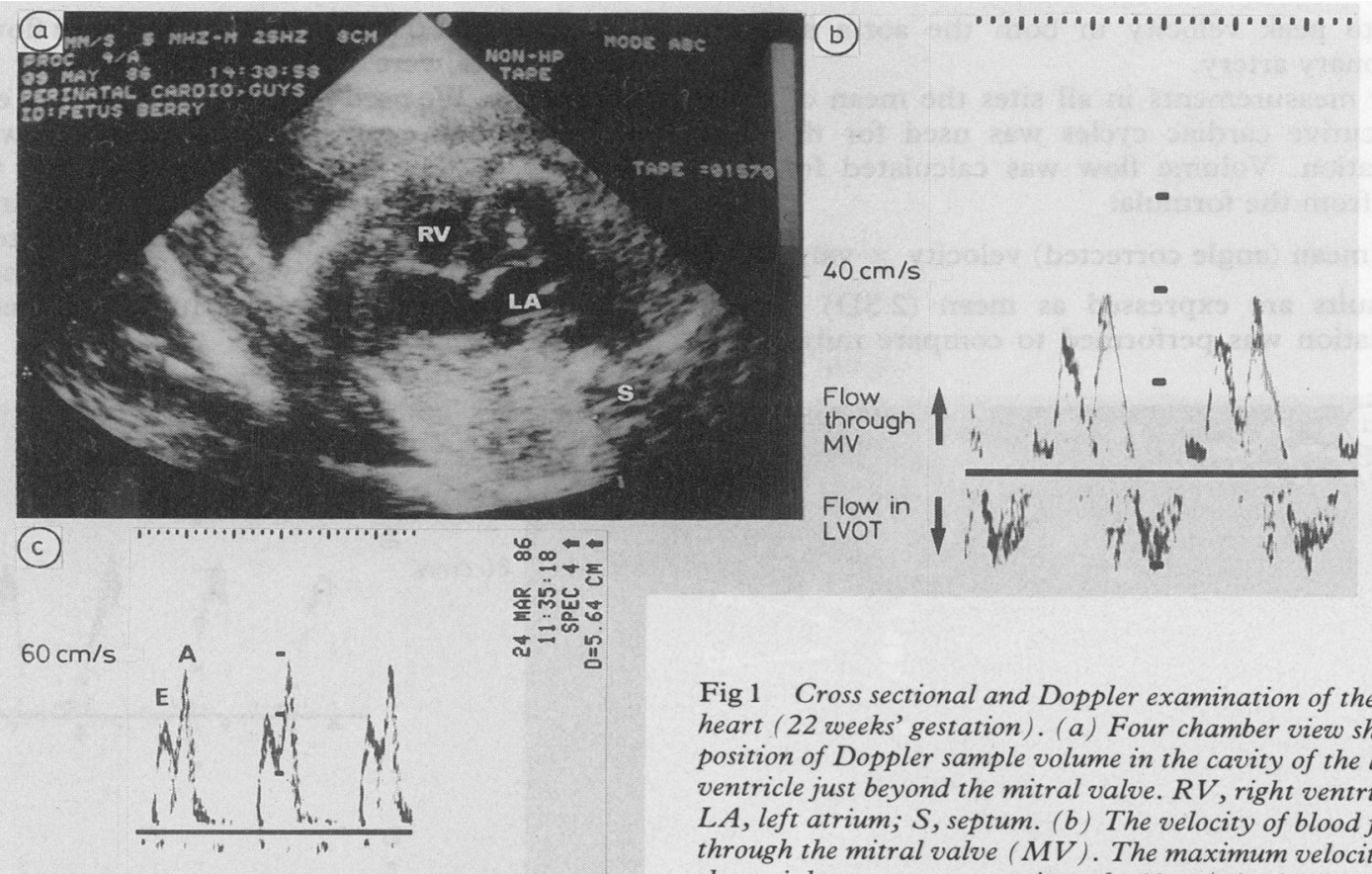

Fig 1 Cross sectional and Doppler examination of the fetal heart (22 weeks' gestation). (a) Four chamber view showing position of Doppler sample volume in the cavity of the left ventricle just beyond the mitral valve. $R V$, right ventricle; $L A$, left atrium; $S$, septum. (b) The velocity of blood flow through the mitral valve ( $M V)$. The maximum velocity of the atrial wave was approximately $40 \mathrm{~cm} / \mathrm{s}$ in this fetus $(22$ weeks' gestation). There is some reverse flow in the left ventricular outflow tract (LVOT) below the zero line. (c) The velocity of blood flow through the tricuspid valve. The atrial wave reached a maximum velocity of $60 \mathrm{~cm} / \mathrm{s}$ in this fetus (22 weeks' gestation).

operator and is automatically corrected in the velocity display. The orifice was measured with callipers on the frozen frame image of the valve under examination. The mitral and tricuspid orifices were measured in diastole in the apical four chamber view. The internal diameter of the aorta and the pulmonary artery were measured, also in diastole, at the tip of the closed arterial valve. For these measurements the origin of the great artery was viewed in the long axis, the ultrasound beam being directed perpendicularly to the walls of the artery whenever possible. All valve orifices were assumed to be circular for the calculation of their cross sectional area.

The maximum velocity through each valve was estimated from the paper recording of the velocity trace. Traces with variations in velocity caused by fetal breathing or other fetal movement were not used for analysis. In acceptable traces the mean temporal velocity was calculated by planimetry of the area under the spectral velocity record throughout the cardiac cycle; this was then divided by the time over which the flow was traced. Flow velocities were traced along the zero line during diastole for the arterial valves and during systole for the atrioventricular valves. Figure 1a shows a four chamber view suitable for atrioventricular valve interrogation. The Doppler sample volume was positioned just beyond the mitral valve orifice, and fig $1 \mathrm{~b}$ shows a representative trace achieved in this position. In early pregnancy the sample overlapped the outflow tract of the left ventricle, hence reverse flow was seen during diastole in this tracing. This was of no importance because it was not included in the flow calculation. This effect was minimised by placing the sample volume as close as possible to the wall of the left ventricle. Figure 1c shows a tracing of tricuspid valve flow velocity. This shows the double peaked velocity trace through both atrioventricular valves. The first peak is the passive filling phase and the second is associated with atrial systole.

Figure 2 shows the sample volume suitably positioned in the pulmonary artery (a) with the resulting velocity trace (b). Figure $3 a$ shows the position of the sample volume in the aorta and fig $3 \mathrm{~b}$ the velocity recorded in this site. The arterial trace showed one velocity peak with a similar acceleration and 
time to peak velocity in both the aorta and the pulmonary artery.

For measurements in all sites the mean of three consecutive cardiac cycles was used for the flow calculation. Volume flow was calculated for each valve from the formula:

$\mathrm{Q}=$ mean (angle corrected) velocity $\times$ valve area.

Results are expressed as mean (2 SD). Linear correlation was performed to compare mitral with aortic flow and tricuspid with pulmonary flow. Similar methods were used to relate velocity to gestational age. We used multiple regression to examine various models expressing the relation between the diameter or flow and the gestational age with or without variation between the mitral and tricuspid valves. The simplest equations which fitted adequately are given. This was repeated for the aortic and pulmonary valves. The null hypothesis was retained when $\mathrm{p}>0.05$.
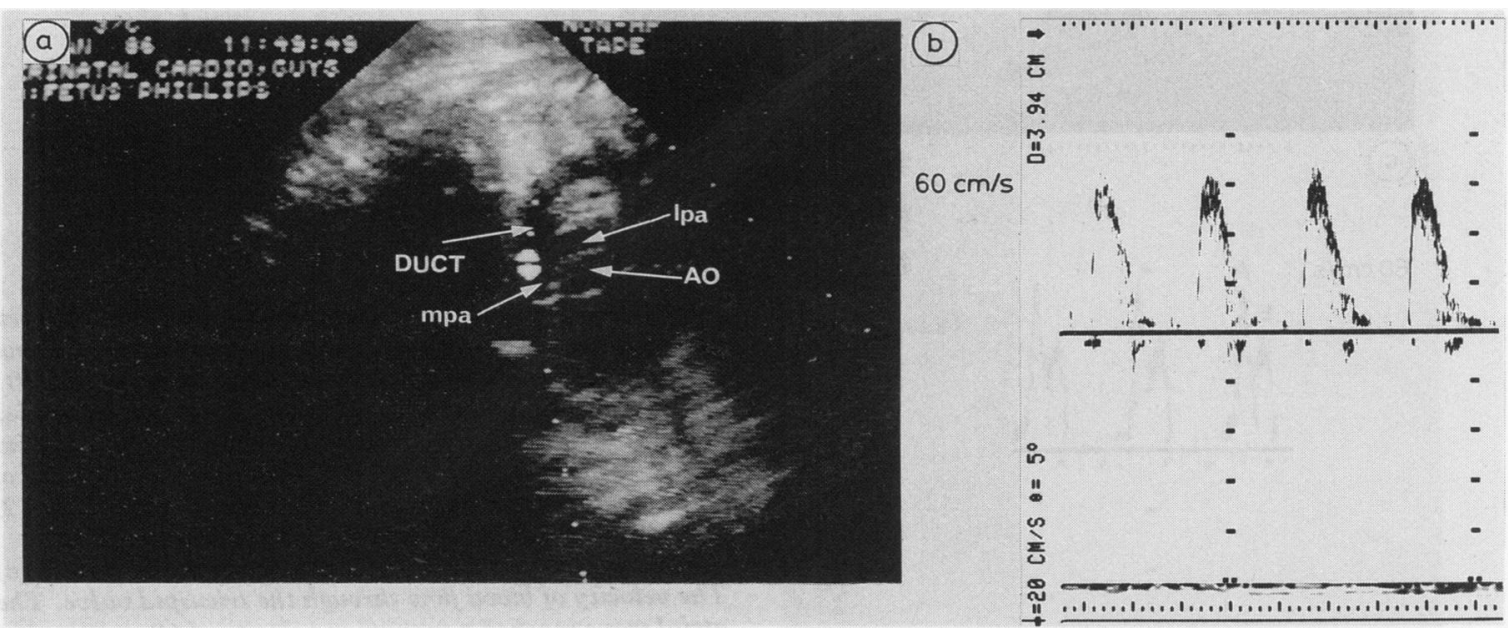

Fig 2 (a) Cross sectional view showing position of the Doppler sample volume in the pulmonary artery. The angle of insonation is very close to the line of blood flow. (b) Pulmonary artery velocity tracing at 24 weeks' gestation. The maximum velocity is approximately $65 \mathrm{~cm} / \mathrm{s}$ in this recording. DUCT, ductus arteriosus; AO, aorta; lpa, left pulmonary artery; mpa, main pulmonary artery.

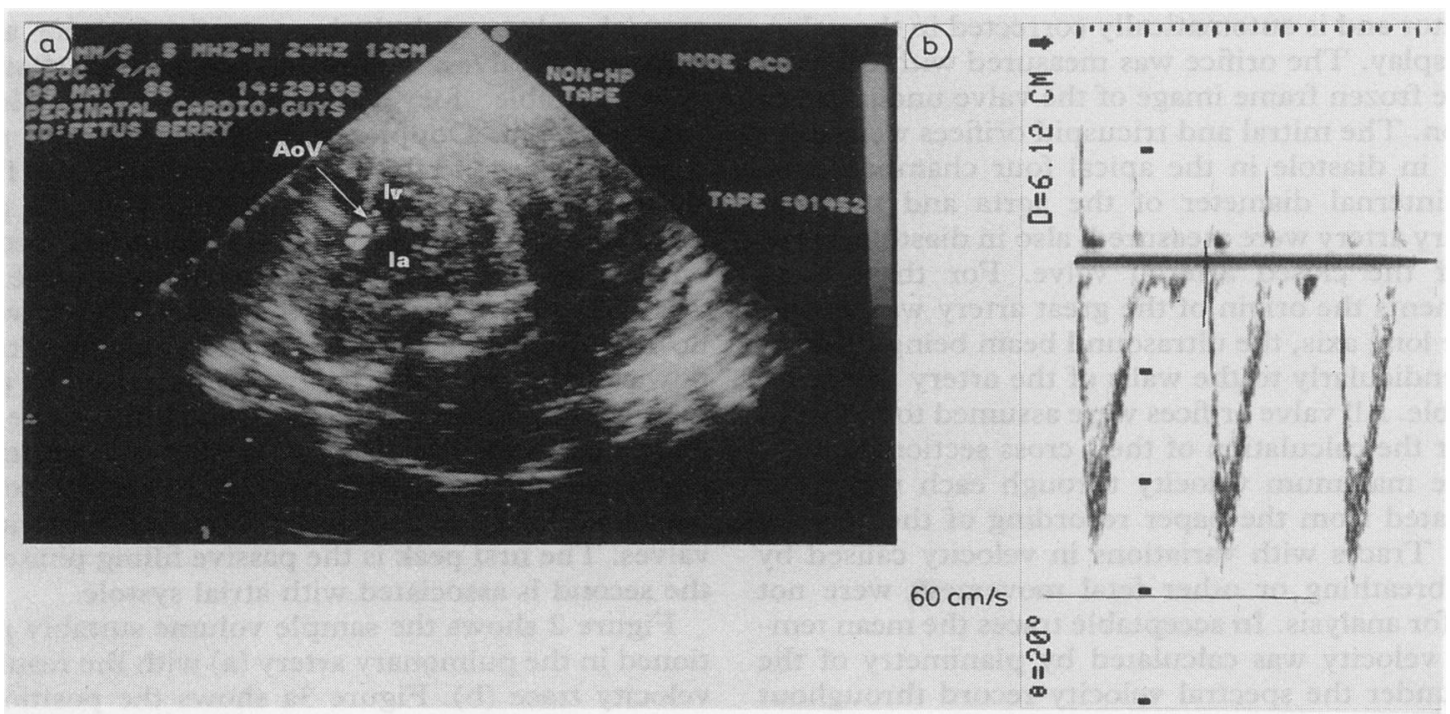

Fig 3 (a) Cross sectional apical view showing position of the Doppler sample volume in the ascending aorta. Imaging from the left ventricular apex gives a good angle of insonation. lv, left ventricle; la, left atrium; AoV, aortic valve. (b) The maximum velocity of aortic blood reached $55 \mathrm{~cm} / \mathrm{s}$ in this fetus (22 weeks' gestation). 


\section{Results}

Adequate images and Doppler traces were obtained in all four sites in 36 fetuses. In the remaining 84 data were obtained from only two or three sites.

The maximum velocity of flow through the atrioventricular valves did not change throughout pregnancy. It remained at a mean of $51.65(19.4) \mathrm{cm} / \mathrm{s}$ through the tricuspid valve and at a mean of $48.49(20.8) \mathrm{cm} / \mathrm{s}$ through the mitral valve. The mean velocity of flow through the tricuspid valve was $13.5(6) \mathrm{cm} / \mathrm{s}$ while that through the mitral was $12.5(5.2) \mathrm{cm} / \mathrm{s}$. Like the maximum velocity, the mean velocity did not change significantly during pregnancy. The differences between the tricuspid and mitral valve values for maximum and mean velocities were not statistically significant. The atrioventricular and arterial valve diameters increased as the heart grew; fig 4 shows the mean values for their dimensions. There was a linear

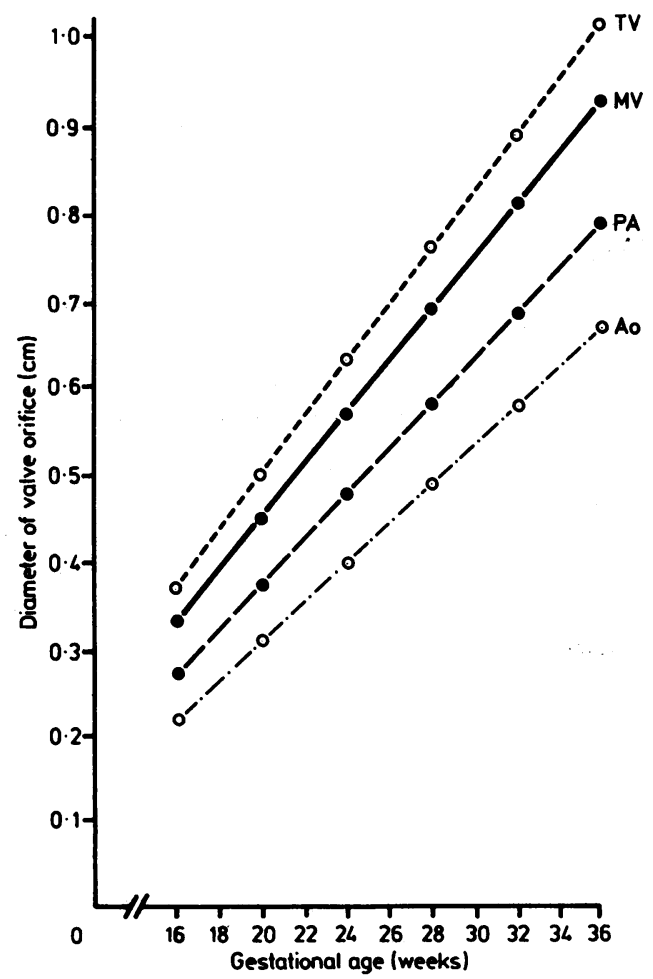

Fig 4 The diameters of all four valves increased throughout pregnancy. At all times the tricuspid (TV) was slightly larger than the mitral (MV) orifice and the pulmonary $(P A)$ orifice was larger than the aortic (Ao) orifice. $M V=$ $-0.146+0.0300$ gestational age; $T V=-0.146+$ 0.0324 gestational age; $A o=-0.138+0.0224$ gestational age; $P A=-0.138+0.0257$ gestational age. relation between all four valves and gestational age. The relevant lines for the atrioventricular values shown in fig 4 have a constant intercept but significantly different slopes $(p<0.001)$. The fit was not significantly better if the intercepts were varied. The same is true of the arterial valves, the pulmonary artery being significantly larger than the aorta $(p<0.001)$.

The maximum velocity in both great arteries ranged from $30 \mathrm{~cm} / \mathrm{s}$ at 16 weeks' gestation to $100 \mathrm{~cm} / \mathrm{s}$ at term. The mean velocity of aortic flow was a mean of $18(8.6) \mathrm{cm} / \mathrm{s}$ and that in the pulmonary artery was $17 \cdot 2(8) \mathrm{cm} / \mathrm{s}$. Mean velocity in the great arteries appeared to increase as pregnancy advanced but this increase was not statistically significant, nor was the difference between the mean velocity in the two great arteries.

Figure 5 shows the calculated volume flows $(\mathrm{ml} / \mathrm{min})$ for each atrioventricular valve and for the arterial valves. The increase in volume flow with increasing gestational age is accounted for by the increase in the size of the valve orifices. The relations between mitral and tricuspid valve flows and gestational age were linear after log transformation, and lines of constant slope but significantly different intercepts $(p<0.001)$ were fitted. The fit was not significantly better if different slopes were fitted to results from the two valves. Figure 5 shows the relation between flow and ges-

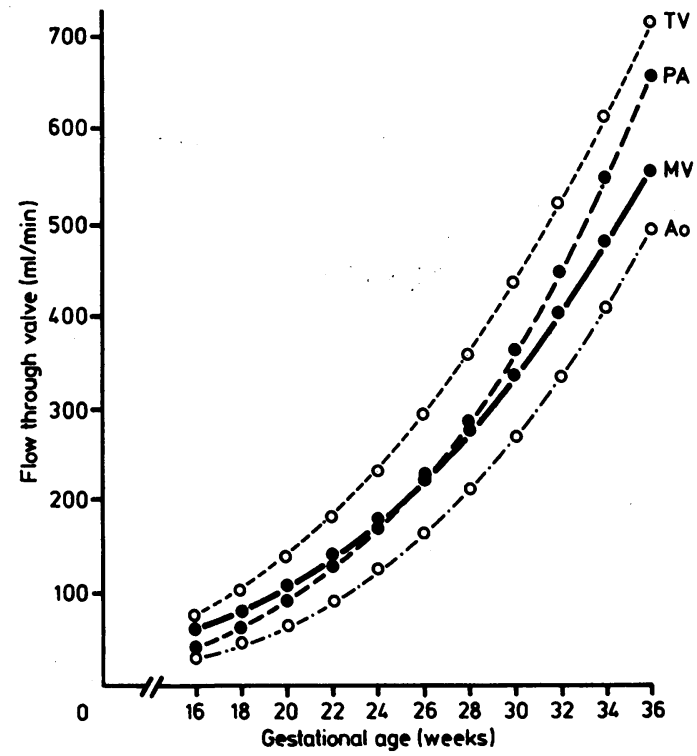

Fig 5 Valve flow increased throughout pregnancy. Between 32 and 34 weeks' gestation the combined ventricular output totalled approximately $1 \mathrm{l} / \mathrm{min} . T V$ flow $=0.03585$

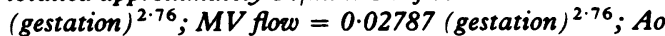
flow $=0.003139$ (gestation $)^{3.34} ; P A$ flow $=0.004201$ (gestation $)^{3 \cdot 34}$. See legend to fig 4 for abbreviations. 
tational age for both these valves on transforming back. A similar transformation was required for the aortic and pulmonary valve flows and the best fit was achieved with two lines of constant slope but significantly different intercepts $(p \leqslant 0.001)$. Again fig 5 shows the relationships on transforming back.

To test the validity of the flow calculations, linear correlations between the mitral and the aortic and the tricuspid and the pulmonary flows were calculated for the 36 fetuses with measurements in all four sites (figs 6 and 7). The data from these 36 fetuses gives information on the relative outputs of the right

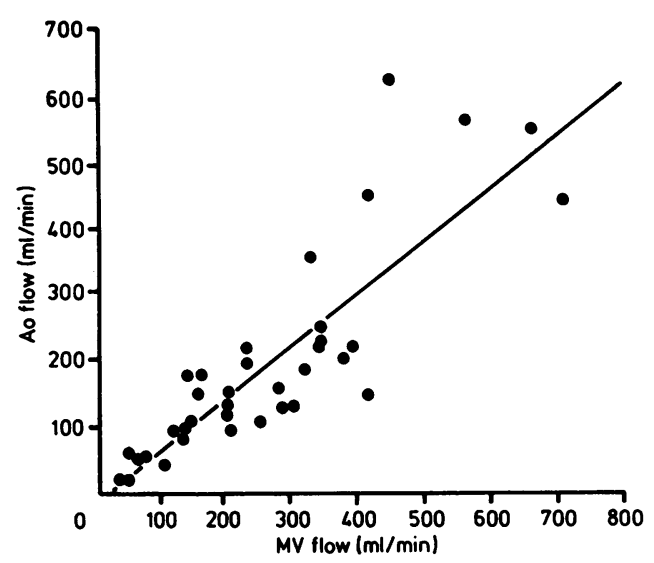

Fig 6 Comparison of estimated aortic (Ao) and mitral valve (MV) blood flows in the same 36 fetuses. The derived regression equation is aortic flow $=-10 \cdot 2+0.82 \times$ mitral flow, with $r=0.874$.

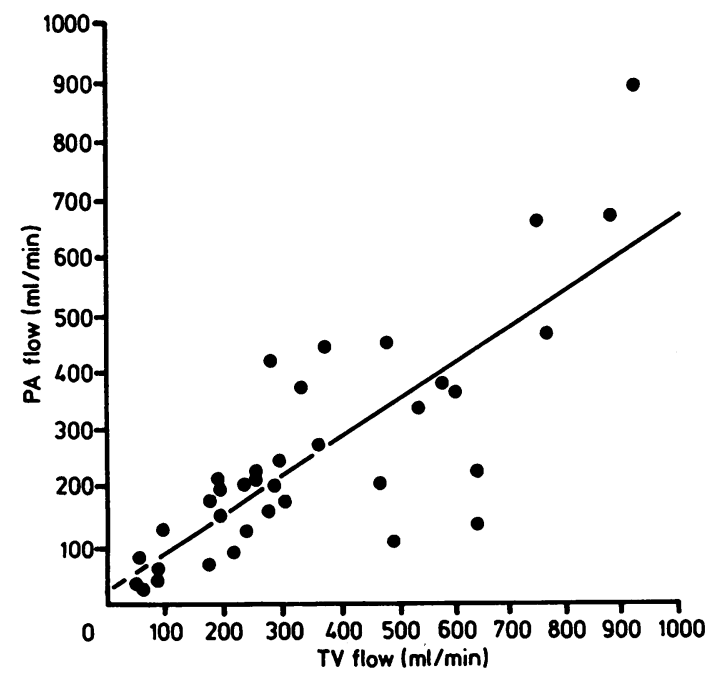

Fig 7 Comparison of pulmonary (PA) and tricuspid valve (TV) blood flows in the same 36 fetuses. The regression equation is pulmonary flow $=21.27+0.68 \times$ tricuspid flow, with $r=0.82$. and left ventricles. When this was estimated from the atrioventricular valve flow the right ventricular output exceeded that of the left ventricle according to the regression equation:

tricuspid flow $=12.56+1.32 \times$ mitral flow;

the ratio of tricuspid to mitral flow ranged from 0.8 to $2 \cdot 2$ (mean $1 \cdot 3$ ). Similarly, pulmonary artery flow exceeded aortic flow. The regression equation was:

pulmonary artery flow $=49 \cdot 25+1.05 \times$ aortic flow; the ratio of pulmonary artery to aortic flow ranged from 0.8 to 2.5 (mean 1.4 ).

\section{Discussion}

The good correlation between atrioventricular valve flow and flow in the corresponding great artery corroborates the validity of Doppler derived volume flow estimates in the human fetus. This is important since, of necessity, the experimental study of the fetal circulation has hitherto been confined to animals. Although the same principles of cardiovascular physiology apply to man and the lamb it is unwise to extrapolate directly from one species to another. The techniques described in this report allow us to gain direct and detailed insights into the cardiovascular performance of the normal intact human fetus.

The measurement of maximum velocity is accurate and reproducible and knowledge of the normal values in the fetus is important when such measurements are made in the presence of structural cardiac abnormality. For example, valve stenosis in prenatal life, as in postnatal life, will increase the maximum velocity across the affected valve.

Although the velocity measurements are accurate, the derived volume flow estimates are subject to error in the measurement and calculation of valve orifice area. The scatter of dimension measurements was greater for the atrioventricular than for the arterial valves. In our experience the decision about which dimension to take as the diameter to measure at the atrioventricular valve is more subjective than the decision at arterial valve. Any error is magnified when the radius is squared in the volume flow calculation. A further possible source of error is the assumption that all four valves are circular in cross section. This is probably true for the arterial valves but may not accurately reflect the geometry of the atrioventricular orifices. For these reasons, and because it is easier, we prefer to take the estimate of flow obtained in the great arteries as the output of that ventricle. Despite these reservations the correlation between atrioventricular valves and arterial valve flows is, as stated above, good. 
Examination of fig 5 shows that the combined ventricular output at 26 weeks' gestation is approximately $450 \mathrm{ml} / \mathrm{min}$, slightly more if atrioventricular valve flow is used and slightly less if arterial valve flow is used. At this gestational age the fetal body weight is approximately $1 \mathrm{~kg}$. Thus the combined ventricular output is in the region of $450 \mathrm{ml} / \mathrm{kg} / \mathrm{min}$. This figure resembles that found by other workers and the values found in fetal lambs. ${ }^{67}$ By 32 weeks' gestation, however, when the mean fetal body weight is $2 \mathrm{~kg}$, the combined ventricular output dropped slightly to $420 \mathrm{ml} / \mathrm{kg} / \mathrm{min}$. There was a clear pattern for the relation between right and left ventricular outputs. In most cases the output of the right ventricle was higher than that of the left, but in less than a quarter of cases the reverse was true.

There is wide diurnal and individual variation in cardiac output in the normal resting subject, and in the fetus it is difficult to be sure that the measurements are made in a resting state. Doppler tracings are very difficult to obtain if there is appreciable movement of the fetal trunk, but finer head or limb movements are impossible to exclude during the study. Such movements could alter the peripheral resistance, and thus the Doppler estimate of mean velocity, and could contribute to the scatter of normal values.

Despite these reservations about the method these data demonstrate that Doppler echocardiography can be used to study human fetal cardiac function. These normal values provide the basis for the interpretation of data obtained from fetuses with abnormalities of pregnancy, structural heart disease, or cardiac arrhythmias.

We thank Mrs Rosemary Brown, senior lecturer in biostatistics, Guy's Hospital, for her work on the statistical analysis.

LDA, SKC, and DCC are supported by the British Heart Foundation; W Al-G is supported by the Iraqi Government; and MT is supported by the Joseph Levy Foundation.

\section{References}

1 Allan LD, Tynan MJ, Campbell S, Wilkinson JL, Anderson RH. Echocardiographic and anatomical correlates in the fetus. Br Heart $J$ 1980;44:444-51.

2 Kleinman CS, Hobbins JC, Jaffe CC, Lynch DC, Talner NS. Echocardiographic studies of the human fetus: prenatal diagnosis of congenital heart disease and cardiac dysrhythmias. Pediatrics 1980;65: 1059-68.

3 Allan LD, Crawford DC, Anderson RH, Tynan MJ. Echocardiographic and anatomical correlations in fetal congenital heart disease. $B r$ Heart $J$ 1984;52: 542-8.

4 Lewis JF, Kuo LC, Nelson JC, Limacher MC, Quinones MA. Pulsed Doppler echocardiographic determination of stroke volume and cardiac output: clinical validation of two new methods using the apical window. Circulation 1984;70:425-31.

5 Meijboom EJ, Horowitz S, Valdes-Cruz LM, Sahn DJ, Larson DF, Lima CO. A Doppler echocardiographic method for calculating volume flow across the tricuspid valve: correlative laboratory and clinical studies. Circulation 1985;71:551-60.

6 Rudolph AM, Heymann MA. The circulation of the fetus in utero. Circ Res 1967;21:163-84.

7 Reed KL, Meijboom EJ, Sahn DJ, Scagnelli SA, Valdes-Cruz LM, Shenker L. Cardiac Doppler flow velocities in human fetuses. Circulation 1986;73: 41-6. 